RELATO dE CASO

CASE REPORT

\title{
Doença de Neuro-Behçet de Início na Infância ${ }^{*}$
}

\author{
Neuro-Behçet's Disease in Childhood-Onset
}

\author{
Teresa Cristina Martins Vicente Robazzi ${ }^{(1)}$, Renata Arruti( ${ }^{(2)}$, Ana Karina Souza ${ }^{(3)}$, Mittermayer B. Santiago ${ }^{(4)}$
}

\section{RESUMO}

Os autores descrevem o caso de um adolescente, que iniciou com quadro clínico de uveíte bilateral e aftas na mucosa oral aos 13 anos de idade. Nesse momento foi estabelecido o diagnóstico de doença de Behçet, evoluindo dois anos após com hemiparesia aguda e deficit motor à direita. A ressonância magnética do crânio evidenciou sinais de vasculite em atividade. O tratamento inicial com ciclofosfamida não se mostrou eficaz, requerendo o uso de clorambucil e posteriormente da azatioprina oral. A doença de Behçet apresenta envolvimento multissistêmico com manifestações oculares, cutânea, ocular, intestinal, articular, vascular, urogenital e neurológica. As manifestações neurológicas têm início mais freqüentemente na população adulta e excepcionalmente na infância e adolescência, representando uma importante causa de invalidez e morte.

Palavras-chave: doença de Behçet, doença de Neuro-Behçet, sistema nervoso central.

\section{INTRODUÇÃO}

A doença de Behçet é uma doença inflamatória crônica e multissistêmica, caracterizada por ulcerações orais e genitais associadas à uveíte, podendo, no entanto, acometer qualquer órgão e sistema ${ }^{(1)}$.

Descrita pela primeira vez por Hipócrates, na Grécia Antiga por volta de 450 a.C., voltou a ser relatada pelo médico Benedictos Adamantiades em Atenas, em 1931. Em 1936, o dermatologista turco, Hulusi Behçet, publicou seus achados como um complexo de sintomas constituindo uma

\begin{abstract}
The authors describe a thirteen years old teenager who had bilateral uveitis and recurrent oral aphthous ulcers. At that moment, Behçet's disease was diagnosed, and after a two-year follow-up, acute right hemiparalysis and motor deficit occured. Magnetic Resonance Imaging of the skull showed signs of active vasculitis. Initial treatment with cyclophosfamide wasn't efficient, requiring the use of clorambucil and, posteriorly, oral azathioprine. Behçet's disease presents with multisystemic manifestations such as ocular, cutaneous, oral, intestinal, articular, vascular, urogenital and neurologic disorders. Neurological manifestations usually begin in adult life and, exceptionally, in childhood and adolescence, representing an important cause of disability and mortality.
\end{abstract}

Keywords: Behçet disease, neuro-Behçet disease, central nervous system.

tríade clínica clássica, que incluía aftas orais recorrentes, úlceras genitais, uveíte com hipópio. Em 1947, no Congresso Internacional de Dermatologia, a doença foi então oficialmente reconhecida, sendo denominada doença de Adamantiades-Behçet, em homenagem a esses dois autores ${ }^{(1)}$.

Casos da doença são descritos em todo o mundo, mas a sua maior prevalência é na antiga rota das sedas, área que se estende do Japão ao sudoeste asiático e países mediterrâneos, sendo na Turquia estimada em 190 por 100.000 habitantes adultos ${ }^{(2)}$. A doença de Behçet é mais freqüentemente descrita na população entre 18-40 anos de idade,

* Trabalho realizado no Serviço de Reumatologia Pediátrica do Hospital São Rafael, Salvador, BA, Brasil. Recebido em 17/06/2004. Aprovado, após revisão, em 10/02/2005.

1. Médica reumatologista pediátrica do Hospital São Rafael, Salvador, BA, Brasil. Mestranda em Medicina Interna pela Universidade Federal da Bahia, Salvador, BA, Brasil.

2. Médica residente de Pediatria do $2^{\circ}$ ano do Hospital São Rafael, Salvador, BA, Brasil.

3. Médica residente de Pediatria do $2^{\circ}$ ano do Hospital São Rafael, Salvador, BA, Brasil.

4. Professor adjunto e doutor da Escola Baiana de Medicina e Saúde Pública, Salvador, BA, Brasil.

Endereço para correspondência: Dra. Teresa Cristina Martins Vicente Robazzi. Rua Leonor Calmon, 44 - sala 1.203, CEP 40280-633. Salvador, BA, Brasil. Tel./fax: 55 (71) 354-3826; E-mail: trobazzi@superig.com.br 
sendo pouco descrita na infầncia e adolescência ${ }^{(3,4)}$. Quando ocorre até a idade de 16 anos é considerada doença de Behçet infanto-juvenil.

Entre as manifestações da doença de Behçet, estão as relacionadas ao sistema nervoso central (SNC), que ocorrem mais freqüentemente na população adulta e excepcionalmente na infầncia e adolescência ${ }^{(5)}$. Constituem a forma mais grave da doença, normalmente se manifestando dois a seis anos após o início da sintomatologia. $\mathrm{O}$ envolvimento do SNC (Neuro-Behçet) é uma das manifestações clínicas iniciais mais freqüentes, com prevalência em torno de $5 \%$ a $7 \%$ da população adulta ${ }^{(6,7,8)}$.

A doença de Neuro-Behçet inclui manifestações clínicas variadas, tais como: meningoencefalites, síndromes piramidais, hipertensão intracraniana benigna, sinais cerebelares e distúrbios psiquiátricos ${ }^{(9,10)}$.

O objetivo do presente estudo foi descrever o caso de um paciente do sexo masculino com diagnóstico de NeuroBehçet aos 15 anos de idade.

\section{RELATO DE CASO}

LDO, 18 anos de idade, sexo masculino, com história de iridociclite de repetição com deficit visual e aftas na mucosa oral desde os 13 anos de idade. Foi diagnosticada uveíte bilateral secundária à doença de Behçet, sendo iniciado o uso de corticoterapia tópica e de ciclosporina oral, na dose de $5 \mathrm{mg} / \mathrm{kg} /$ dia, fracionado em duas doses diárias com tempo total de uso de 1 ano e 6 meses. Evoluiu sem novas queixas clínicas até a idade de 15 anos, quando apresentou quadro agudo de hemiparesia à direita, disartria, deficit motor à direita com fraqueza muscular grau IV, segundo os critérios do Medical Research Council, liberação piramidal e Babinski à direita, com as funções mentais preservadas. A ressonância magnética (RM) do crânio evidenciou na seqüência Flair axial e sagital ponderada em T2, hiperintensidade de sinal, acometendo cápsulas internas e núcleos pálidos de ambos os lados, bem como tálamo esquerdo (Figura 1). Foi feito o diagnóstico de Neuro-Behçet e iniciada pulsoterapia com metilprednisolona por cinco dias, com manutenção de prednisona oral (30mg/dia). Dois dias após a alta hospitalar, apresentou recidiva do quadro clínico, quando então foram associadas ao corticóide, sessões de pulsoterapia mensais com ciclofosfamida venosa na dose de $500 \mathrm{mg} / \mathrm{m}^{2}$, totalizando seis meses de uso. Após quatro e seis meses, respectivamente, apresentou mais duas recidivas do quadro clínico, apresentando-se clinicamente com cefaléia intensa, vômitos, sonolência, amnésia lacunar e piora significativa do deficit motor à direita. Optou-se por introduzir clorambucil na dose de $0,1 \mathrm{mg} / \mathrm{kg} / \mathrm{dia}$, fracionado em duas doses, associado ao corticóide.

A pesquisa dos auto-anticorpos (anti-DNA ds, FAN, antiSM, anti-Ro, anti-La, ANCA e anti-RNP) foi realizada, objetivando o diagnóstico diferencial com outras vasculites, porém, os resultados foram negativos. A avaliação laboratorial dos demais órgãos e sistemas foi normal. Não foi realizada pesquisa de HLA-B51, por questões operacionais.

O paciente evoluiu com quadro clínico mantido, sem novas recidivas clínicas, sendo optado, após extensa revisão de literatura e poucos dados referentes ao tratamento da Neuro-Behçet nessa faixa etária, pela substituição do clorambucil (após seis meses de uso) pela azatioprina, visando prevenir possíveis efeitos adversos relacionados à malignidade e infertilidade.

No momento atual, o paciente mantém seqüelas neurológicas e visuais, porém controladas e estabilizadas. Em uso de azatioprina ( $1 \mathrm{mg} / \mathrm{kg} /$ dia $)$ e baixas doses de prednisona oral (10 mg).

\section{DISCUSSÃO}

A doença de Behçet é uma desordem inflamatória multissistêmica crônica, caracterizada por uma disfunção vascular imunológica que afeta principalmente pele, mucosas, SNC, olhos e articulações. Sua etiologia e patogênese são desconhecidas, estando várias hipóteses implicadas, tais como a participação de fatores imunológicos, infecciosos e ambientais (pesticidas, metais pesados) em indivíduos geneticamente predispostos, que apresentam o alelo HLA-B51, principalmente seu subcomponente ${ }^{*} 01^{(11)}$.

Na população adulta, segundo a literatura, há predominância do sexo masculino sobre o feminino ${ }^{(12)}$, ocorrendo mais freqüentemente entre 18 e 40 anos de idade. Na infância, a doença não é freqüente e é caracterizada por uma baixa incidência de lesão ocular e neurológica e alta incidência de envolvimento intestinal ${ }^{(13,14)}$. Esse paciente não apresentou comprometimento cutâneo e intestinal, sendo os componentes marcantes de sua doença, o ocular e o neurológico. É questionado se o diagnóstico da doença na infầncia e adolescência não é subestimado, uma vez que os dados clínicos propostos pelo Study Group for Behçet's Disease (1990), não são patognomônicos e nem sempre estão presentes no início da doença, o que pode dificultar e retardar o diagnóstico ${ }^{(15)}$. É descrita uma tendência cronológica ao aparecimento dos seguintes sintomas: ulcerações orogenitais, lesões dermatológicas, alterações 

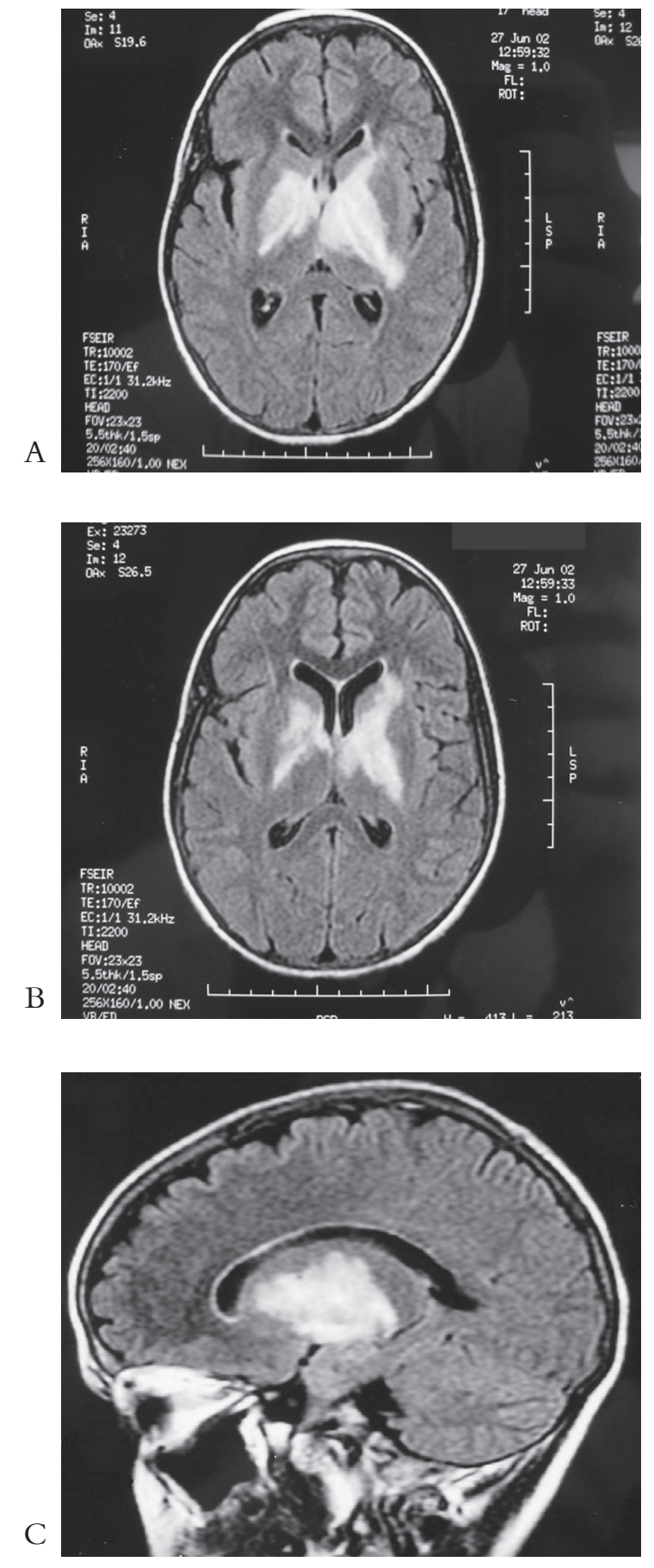

Figura 1 - Paciente de 16 anos de idade, com diagnóstico de doença de Behçet aos 13 anos, evoluindo aos 15 anos com hemiparesia à direita, disartria, deficit motor à direita grau IV, liberação piramidal e Babinski à direita, com as funções mentais preservadas. A e B - Ressonância magnética (RM) de crânio na seqüência Flair axial ponderada em T2: hiperintensidade de sinal, acometendo cápsulas internas e núcleos pálidos de ambos os lados, bem como tálamo esquerdo. C - RM de crânio na sequiência Flair sagital ponderada em T2: hiperintensidade de sinal, acometendo cápsula interna, núcleo pálido, bem como tálamo esquerdo oculares e, após alguns anos da doença, o surgimento de manifestações vasculares, intestinais e neurológicas. No entanto, um início da doença marcado com lesões graves pode ocorrer. $\mathrm{Na}$ ausência do envolvimento do SNC e da presença de complicações vasculares, o prognóstico geralmente é bom ${ }^{(3)}$.

$\mathrm{O}$ primeiro sintoma em adultos pode ser neurológico em $5 \%$ a $7 \%$ dos casos, mas no curso da doença a prevalência de Neuro-Behçet pode variar de 10\% a 50\%. Em crianças, sintomas neurológicos como apresentação inicial são mais raros, havendo relato na literatura de duas crianças iniciando a doença com sintomas de meningoencefalite e pseudotumor cerebral ${ }^{(9)}$.

A prevalência de Neuro-Behçet na infância, segundo a literatura, se situa em torno de $5 \%{ }^{(9)}$, podendo ocorrer meningoencefalite, síndrome de pseudotumor cerebral, síndrome mental orgânica (insônia, perda de memória, apatia, agitação psicomotora, mania, delírio), distúrbio cerebelar, neuropatia periférica e cefaléia recorrente. O curso clínico pode ser recorrente/intermitente, progressivo, primário ou secundário e ainda subclínico, com presença de lesões assintomáticas ${ }^{(16)}$. A este último grupo e à presença de cefaléia em pacientes com sintomas sugestivos de doença de Behçet, deve-se atentar a possibilidade de envolvimento do SNC.

A RM do crânio é o método mais sensível para o estudo de Neuro-Behçet com envolvimento parenquimatoso, devendo ser incluído o diagnóstico diferencial com outras vasculites, doenças inflamatórias e esclerose múltipla ${ }^{(17)}$.

O tratamento é direcionado ao tipo de manifestação clínica apresentada. É de caráter imediato, nos casos de comprometimento do trato gastrintestinal, do sistema nervoso central e do envolvimento de grandes vasos, necessitando altas doses de corticóides e imunossupressores.

A relação do uso de ciclosporina A para o tratamento da doença ocular inflamatória, com o desenvolvimento de manifestações neurológicas, seja por um papel neurotóxico da droga seja pela aceleração do desenvolvimento da doença, é sugerida por alguns autores ${ }^{(8,18,19)}$. O paciente em questão fez uso de ciclosporina A para o tratamento da uveíte, por um período de dois anos antecedendo o início das manifestações neurológicas.

Os agentes citotóxicos (azatioprina, ciclofosfamida, metotrexato, clorambucil), associados aos corticosteróides, são utilizados nos casos de maior gravidade, como nos casos de enterite de delgado grave e vasculite do $\mathrm{SNC}^{(9,13,14)}$.

Sendo assim, chamamos a atenção para a possibilidade de envolvimento neurológico na doença de Behçet juvenil, uma vez que representa importante causa de mortalidade, invalidez e seqüelas neurológicas. 


\section{REFERÊNCIAS}

1. Behçet H: Über rezidivierende Aptose durch ein verursachte Geschwüre am Mund, am Auge und na den Genitalien. Dermatol Wochenschr 105: 1152 -7, 1937.

2. Sakane T, Takeno M, Suzuki N, et al: Behçet disease. N Engl J Med 341: 1284-91, 1999.

3. Eldem B, Onur C, ÖzmS: Clinical features of pediatric Behçet's disease. J Pediatr Ophthmal Strabismus 35: 459-611, 1998.

4. Yamazaki S, Koyano T: A case of Pediatric Behçet's disease with intestinal involvement. J dermatol 26: 160-3, 1999.

5. Koné-Paut I, Bernard JL: Epidemiology of Behçet's disease in children: A French nationwide survey. Arthritis Rheum 35 (Suppl): 190, 1992.

6. Serdaroglu P: Behçet's disease and nervous system. J Neurol 245 197-205, 1988.

7. Kocer N, Islak C, Silva A, et al: CNS involvement in Neuro-Behçet Syndrome: An MR Study. AJNR 20: 1015-24, 1999.

8. Kotake S, Ichiishi A, Kosaka S, Yoshikawa K, Minagawa R, Matsuda $\mathrm{H}$ : Low dose cyclosporin treatment for ocular lesions of Behcet's disease. Acta Soc Ophthamol Jpn 96: 1290-4, 1992.

9. Koné-Paut I, ChabrolB, Riss JM, Mancini J: Neurologic Onset of Behçet's Disease: a Diagnostic Enigma in Childhood. J Child Neurol 12: 237-41, 1997.

10. Inaba G: Clinical features of Neuro-Behçet's syndrome. In Lehner T, Barnes CG (eds): Recent Advances in Behçets Disease, London Royal Society of Medicine, p. 235-46, 1988.
11. Takeno M, Kariyone A, Yamashita N, et al: Excessive function of peripheral blood neutrophils from patients with Behçet's disease and from HLA-B51 transgenic mice. Arthritis Rheum 38: 426-33, 1995.

12. Chajek T, Fainaru M: Behçet's disease report of cases and a review of the literature. Medicine 54: 179-96, 1995.

13. Kim DK, Ghang SN, Bang Dongsik, et al: Clinical Analisys of 40 Cases of Chilhood - Onset Behçet's Disease. Pediatric Dermatology 11: 95-101, 1992.

14. Ke Y, Hwang K, Lee C: Behçet's Disease in Childhood: Report of One Case. Acta Pediatr Tw 44: 292-6, 2003.

15. Wechsler B, Davatchi F, Mizushima Y, et al. International Study Group for Behçet's disease. Criteria for diagnosis of Behçet's disease. Lancet 335: 1078-80, 1990.

16. Akman D, Serdaroglu P, Tasci B, et al: Clinical patterns of neurological involvement in Behçet's disease: evaluation of 200 patients. Brain 122: 2171-81, 1999.

17. Cohan O, Bahar S, Akman-Demir G, et al: Masked Assessment of MRI findings:is it possible to differentiate Neuro-Behçet disease from other central nervous system diseases? Neuroradiology 41: 255-60, 1999.

18. Kato Y, Numanga J, Kato S, et al: Central nervous system symptoms in a population of Behçet's disease patients with refractory uveitis treated with cyclosporine A Clinical and Experimental Ophthalmology 29: 335-6, 2001.

19. Kotake S, Higashi K, Yoshikawa K, et al: Central Nervous System Symptoms in Patients with Behçet Disease Receiving Cyclosporine Therapy. Ophthalmology 106: 586-9, 1999. 